\title{
DIRICHLET FORMS METHODS : AN APPLICATION TO THE PROPAGATION OF THE ERROR DUE TO THE EULER SCHEME
}

\author{
Nicolas Bouleau \\ bouleau@enpc.fr \\ Ecole des Ponts, ParisTech
}

\begin{abstract}
We present recent advances on Dirichlet forms methods either to extend financial models beyond the usual stochastic calculus or to study stochastic models with less classical tools. In this spirit, we interpret the asymptotic error on the solution of an sde due to the Euler scheme (Kurtz and Protter [Ku-Pr-91a]) in terms of a Dirichlet form on the Wiener space, what allows to propagate this error thanks to functional calculus.
\end{abstract}

Keywords : squared field operator, Wiener space, density, Dirichlet process, stochastic differential equation, Dirichlet form, error.

\section{Introduction}

Considering a Dirichlet form amounts to consider a strongly continuous symmetric contraction semi-group on an $L^{2}$-space which possesses in addition the property of being positive on positive functions (cf. [Fu-Os-Ta-94], [Bo-Hi-91 ], [Ma-Rö-92]). It is a particular case of Markovian potential theory, with several special features due to the use of Hilbertian techniques and to the fact that positivity and contraction properties extend to infinite dimensional framework thanks to Fatou's lemma in measure theory. Many Dirichlet structures are constructively obtained on the Wiener space and on the fundamental spaces of probability theory (Poisson space, Monte Carlo space) which may be thought as hypotheses in order to study error propagation through stochastic models (cf. [Bou-03b]).

Since the discovery by M. Fukushima, at the end of the seventies, that Dirichlet forms allow to extend the stochastic calculus to processes which are not semi-martingales (cf. [Fuk-80]) a lot of works have been developed in this direction, even beyond the Dirichlet forms framework. To this extend we quote the approach to time-dependent Dirichlet forms developed by Oshima [Osh-92] and the more recent approach of Stannat [Sta-99] and Trutnau [Tru-00] about a new theory of generalized Dirichlet forms. As in finance the heart of the complete market property and more generally of the portfolio management is the stochastic integral, a particular interest has been devoted to methods giving rise to new stochastic integrals.

We shall give, at first, a short outlook on recent results related to Dirichlet forms and connected with financial motivations. We include some Malliavin calculus approaches when they amount to the use of the Ornstein-Uhlenbeck structure on the Wiener space. After recalling, in a second part, the main properties of Dirichlet forms and the interpretation of the functional calculus on the squared field operator in terms of error propagation, we focuse, in a third part, on the question of the asymptotic error due to the resolution of a stochastic differential equation by the Euler scheme. We show that the asymptotic 
to propagate the error on the example of a level volatility model for pricing and hedging procedures. We put the general question of the validity of such a propagation as an asymptotic calculus principle, and we give partial arguments for this principle.

\section{Some recent works.}

First must be mentionned the idea of using Malliavin's integration by parts technique to speed up the computation of the Greeks or other quantities in finance. After the collective papers of Fournier and al [Fo-La-Le-Li-To-99] [Fo-La-Le-Li-01], improvements have been brought to complex options [Go-Ko-01] and to the more general question of the sensitivity to some parameters with the aim of calibration of a model. As integration by parts formulae are available in more general Dirichlet forms situations than the OrnsteinUhlenbeck structure on the Wiener space (cf. [Bou-03b] Chapter V), the same approach may be performed for instance on the Poisson space for studying models with jumps [El-Pr-04].

One of the first success of Malliavin calculus was about proving existence of densities for solutions of sde's with smooth coefficients and Dirichlet forms methods have been able to extend such results to the case of Lipschitz coefficients [Bo-Hi-91]. Several authors remarked that these tools give also means of improving the computation of densities and establishing estimates for the laws of random variables with some regularity assumption. Let us quote ([Ko-Pe-02] , [Ca-Fe-Nu-98], [Bo-Ek-To-04], [Bou-05b]) whose results aren't limited to applications in finance. With suitable hypotheses it is possible, to get explicit closed formulae for the density even with some liberty in the choice of a weight function allowing an optimization for Monte Carlo simulation.

After the classical works of M. Fukushima and Y. Le Jan on stochastic calculus for additive functionals of symmetric Markov processes associated with a Dirichlet form [LeJ-78] the role of past and future $\sigma$-algebras have been clarified by Lyons and Zheng (cf [LyZh-98] [Tru-00]) and the main current of research, in order to leave the semi-martingale context, starts with the abstract definition of a Dirichlet process as sum of a local martingale and a process with zero quadratic variation (see [Föl-80]). Because the quadratic variation, as formal Dirichlet form, does not possess the closedness property, the Dirichlet form framework is replaced here by functional analytic arguments. The integral is generally defined by a discretization procedure (cf. [Föl-81], [Bou-85], [Fö-Pr-Sh-95] ) or by a regularization procedure (see [Ru-Va-95], [Ru-Va-96]). These ways have been deepened with the center example of the fractional Brownian motion (cf. [Er-Ru-98], [Zah-98], [Fe-LaP-99], [Al-Ma-Nu-00], [Ru-Va-00], [Gr-No-03], [Gr-Ru-Va-03]). The connections of these works with finance are many : attempting to generalize Girsanov theorem in order to define martingale measures by erasing more general drifts and using generalized stochastic integration (forward, symmetric and backward integrals) in order to deal with exotic models (cf [Fl-Ru-Wo-03]). About "inside trading" and the use of forward integral it is worth to quote [Le-Na-Nu-03].

At last, let us mention some uses of Dirichlet forms or Malliavin calculus to deal with processes with jumps by equipping the general Poisson space with a differential structure (cf. [De-Gr-Po-99], [Me-Pr-03], ) and the forthcoming book of P. Malliavin and A. Thalmaier [Ma-Th-05] whose last chapter is devoted to calculus of variations for markets with jumps, the other ones being strongly related with the above topics.

\section{Dirichlet forms theory seen as error propagation theory.}


we consider an erroneous quantity with a centered small error and apply to it a non linear map, we observe by an easy Taylor expansion argument that

- the error is no more centered in general : a bias appears

- the variance transmit with a first order calculus.

Now if we go on, applying anew several non-linear applications

- the variances and the biases keep (except special cases) the same order of magnitude

- the biases follow a second order differential calculus involving the variances.

With natural notation

$$
\begin{gathered}
\sigma_{n+1}^{2}=f_{n+1}^{\prime 2}\left(x_{n}\right) \sigma_{n}^{2} \\
\operatorname{bias}_{n+1}=f_{n+1}^{\prime}\left(x_{n}\right) \operatorname{bias}_{n}+\frac{1}{2} f_{n+1}^{\prime \prime}\left(x_{n}\right) \sigma_{n}^{2} .
\end{gathered}
$$

The first relation has been discovered, even in several dimension with correlation between the errors, by Gauss at the beginning of the nineteenth century.

From this observation, in order to represent the propagation of small errors we may consider that

1) the variances of errors have to be managed by a quadratic first order differential operator $\Gamma$,

2) the biases of errors have to be represented by a linear second order differential operator $A$,

the propagation of errors being the result of the following change of variable formulae :

$$
\begin{gathered}
\Gamma\left[F\left(X_{1}, \ldots, X_{m}\right), G\left(Y_{1}, \ldots, Y_{n}\right)\right]=\sum_{i j} F_{i}^{\prime}\left(X_{1}, \ldots, X_{m}\right) G_{j}^{\prime}\left(Y_{1}, \ldots, Y_{n}\right) \Gamma\left[X_{i}, Y_{j}\right] \\
A\left[F\left(X_{1}, \ldots, X_{m}\right)\right]=\sum_{i} F_{i}^{\prime}\left(X_{1}, \ldots, X_{m}\right) A\left[X_{i}\right]+\frac{1}{2} \sum_{i j} F_{i j}^{\prime \prime}\left(X_{1}, \ldots, X_{m}\right) \Gamma\left[X_{i}, X_{j}\right] .
\end{gathered}
$$

Because of these propagation rules for the variances and the biases, little errors may be thought as second order vectors. This old notion of differential geometry has been revived at the beginning of the eighties by the study of semi-martingales on manifolds (cf. [Sch-82] [Mey-82] [Eme-89]).

Now, instead of germs of semi-martingales and second order vectors, we will use Dirichlet forms, carré du champ and generator. There are two important reasons for this, that I shall give just after recalling some definitions and examples.

Definition An error structure is a term

$$
S=(\Omega, \mathcal{A}, \mathbb{P}, \mathbb{D}, \Gamma)
$$

where $(\Omega, \mathcal{A}, \mathbb{P})$ is a probability space, and:

(1) $\mathbb{D}$ is a dense subvector space of $L^{2}(\Omega, \mathcal{A}, \mathbb{P})$ (also denoted $L^{2}(\mathbb{P})$ );

(2) $\Gamma$ is a positive symmetric bilinear application from $\mathbb{D} \times \mathbb{D}$ into $L^{1}(\mathbb{P})$ satisfying "the functional calculus of class $\mathcal{C}^{1} \cap$ Lip". This expression means

$$
\forall u \in \mathbb{D}^{m}, \quad \forall v \in \mathbb{D}^{n}, \quad \forall F: \mathbb{R}^{m} \rightarrow \mathbb{R}, \quad \forall G: \mathbb{R}^{n} \rightarrow \mathbb{R}
$$

with $F, G$ being of class $\mathcal{C}^{1}$ and Lipschitzian, we have $F(u) \in \mathbb{D}, G(v) \in \mathbb{D}$ and 
(3) the bilinear form $\mathcal{E}[u, v]=\frac{1}{2} \mathbb{E}[\Gamma[u, v]]$ is "closed". This means that the space $\mathbb{D}$ equipped with the norm

$$
\|u\|_{\mathbb{D}}=\left(\|u\|_{L^{2}(\mathbb{P})}^{2}+\mathcal{E}[u, u]\right)^{1 / 2}
$$

is complete.

If, in addition

(4) the constant function 1 belongs to $\mathbb{D}$ (which implies $\Gamma[1]=0$ by property 2), we say that the error structure is Markovian.

We will always write $\mathcal{E}[u]$ for $\mathcal{E}[u, u]$ and $\Gamma[u]$ for $\Gamma[u, u]$.

With this definition, the form $\mathcal{E}$ is known in the literature as a local Dirichlet form on $L^{2}(\Omega, \mathcal{A}, \mathbb{P})$ that possesses a "squared field" operator (or a "carré du champ" operator) $\Gamma$. These notions are usually studied on $\sigma$-finite measurable spaces. We limit ourselves herein to probability spaces both for the sake of simplicity and because we will use images and products of error structures.

Under very weak additional assumptions, to an error structure (also to a Dirichlet form on a $\sigma$-finite measurable space) a strongly-continuous contraction semigroup $\left(P_{t}\right)_{t \geq 0}$ on $L^{2}(\mathbb{P})$ can be uniquely associated, which is symmetric with respect to $\mathbb{P}$ and sub-Markov. This semigroup has a generator $(A, \mathcal{D} A)$, a self-adjoint operator that satisfies:

$$
A[F(u)]=\sum_{i} \frac{\partial F}{\partial x_{i}}(u) A\left[u_{i}\right]+\frac{1}{2} \sum_{i, j} \frac{\partial^{2} F}{\partial x_{i} \partial x_{j}}(u) \Gamma\left[u_{i}, u_{j}\right] \mathbb{P} \text {-a.s. }
$$

for $F: \mathbb{R}^{m} \rightarrow \mathbb{R}$ of class $\mathcal{C}^{2}$ with bounded derivatives and $u \in(\mathcal{D} A)^{m}$ such that $\Gamma\left[u_{i}\right] \in$ $L^{2}(\mathbb{P})$.

Example 1.(Ornstein-Uhlenbeck structure in dimension 1)

$\Omega=\mathbb{R}, \quad \mathcal{A}=$ Borel $\sigma$-field $\mathcal{B}(\mathbb{R}), \quad \mathbb{P}=\mathcal{N}(0,1)$ reduced normal law, $\quad \mathbb{D}=$ $H^{1}(\mathcal{N}(0,1))=\left\{u \in L^{2}(\mathbb{P}), u^{\prime}\right.$ in the distribution sense belongs to $\left.L^{2}(\mathbb{P})\right\}, \quad \Gamma[u]=u^{\prime 2}$, then $\left(\mathbb{R}, \mathcal{B}(\mathbb{R}), \mathcal{N}(0,1), H^{1}(\mathcal{N}(0,1)), \Gamma\right)$ is an error structure with generator

$$
\begin{gathered}
\mathcal{D} A=\left\{f \in L^{2}(\mathbb{P}): f^{\prime \prime}-x f^{\prime} \text { in the distribution sense } \in L^{2}(\mathbb{P})\right\} \\
A f=\frac{1}{2} f^{\prime \prime}-\frac{1}{2} I \cdot f^{\prime}
\end{gathered}
$$

where $I$ is the identity map on $\mathbb{R}$.

Example 2. (Monte Carlo structure in dimension 1)

$\Omega=[0,1], \quad \mathcal{A}=$ Borel $\sigma$-field, $\quad \mathbb{P}=$ Lebesgue measure, $\quad \mathbb{D}=\left\{u \in L^{2}([0,1], d x)\right.$ the derivative $u^{\prime}$ in the distribution sense over ]0,1[ belongs to $\left.L^{2}([0,1], d x)\right\}, \quad \Gamma[u]=u^{\prime 2}$. Example 3. (Friedrich extension of a symmetric operator)

Let $D$ be a connected open set in $\mathbb{R}^{d}$ with unit volume. Let $\mathbb{P}=d x$ be the Lebesgue measure on $D$. Let $\Gamma$ be defined on $\mathcal{C}_{k}^{\infty}(D)$ via

$$
\Gamma[u, v]=\sum_{i j} \frac{\partial u}{\partial x_{i}} \frac{\partial v}{\partial x_{j}} a_{i j}, \quad u, v \in \mathcal{C}_{k}^{\infty}(D)
$$

where the functions $a_{i j}$ satisfy

$$
a_{i j} \in L_{l o c}^{2}(D) \quad \frac{\partial a_{i j}}{\partial x_{k}} \in L_{l o c}^{2}(D) \quad i, j, k=1, \ldots, d,
$$


then the pre-structure $\left(D, \mathcal{B}(D), \mathbb{P}, \mathcal{C}_{k}^{\infty}(D), \Gamma\right)$ is closable.

Let us now come back to the question of using Dirichlet forms instead of second order vectors as germs of semi-martingales.

The first reason is the closedness property. That gives all the power to this theory. It is similar to $\sigma$-additivity in probability theory. Without the closedness property, we have an apparently more general framework (as additive set functions are more general than $\sigma$-additive ones), but it becomes impossible to say anything on objects which are defined by limits, error propagation is limited to explicit closed formulae. Instead, this closedness property allows to extend error calculus to infinite dimensional frameworks and to propagate errors through typically limit objects as stochastic integrals. As David Hilbert argued against intuitionists, more theorems is better. The philosopher Carl Popper made this mistake about axiomatization of probability theory emphasing that his system (without $\sigma$-additivity) was more general than that of Kolmogorov (with $\sigma$-additivity).

What is particularly satisfying is that this closedness property is preserved by products. Any countable product of error structures is an error structure and the theorem on products (cf. [Bou-03b]) gives explicitely the domain of the new $\Gamma$ operator. Starting with the Ornstein-Uhlenbeck structure in dimension one, the infinite product of this structure by itself gives the Ornstein-Uhlenbeck structure on the Wiener space. Less surprisingly, the image of an error structure, defined in the most natural way, is still an error structure, as an image of a probability space by a measurable map is still a probability space.

The second reason is related to simplicity. Let us come back to the first remark at the beginning of this part. We said that starting with a centered error, centeredness is lost after a non linear map. But what is preserved by image? Which property is an invariant? It is the global property of symmetry with respect to a measure. If the operators describing the error are symmetric with respect to some measure, the image of the error has still this symmetry with respect to the image measure. Centeredness is nothing but symmetry with respect to Lebesgue measure (not a probability measure, a $\sigma$-finite measure but this doesn't matter really here).

\section{The gradient and the sharp (\#).}

In addition to the operators $\Gamma$ and $A$ we will need the notion of gradient which is a linear (Hilbert valued) version of the standard deviation of the error.

Definition. Let $\mathcal{H}$ be a Hilbert space. A linear operator $D$ from $\mathbb{D}$ into $L^{2}(\mathbb{P}, \mathcal{H})$ is said to be a gradient (for $S$ ) if

$$
\forall u \in \mathbb{D} \quad \Gamma[u]=<D u, D u>_{\mathcal{H}} .
$$

A gradient always exists as soon the space $\mathbb{D}$ is separable. It satisfies necessarily the chain rule :

Proposition Let $D$ be a gradient for $S$ with values in $\mathcal{H}$. Then $\forall u \in \mathbb{D}^{n}, \forall F \in \mathcal{C}^{1} \cap$ $\operatorname{Lip}\left(\mathbb{R}^{n}\right)$,

$$
D[F \circ u]=\sum_{i=1}^{n} \frac{\partial F}{\partial x_{i}} \circ u D\left[u_{i}\right] \quad \text { a.e. }
$$

What we denote by the sharp \# is a special case of gradient operator when $\mathcal{H}$ is chosen to be $L^{2}(\hat{\Omega}, \hat{\mathcal{A}}, \hat{\mathbb{P}})$ where $(\hat{\Omega}, \hat{\mathcal{A}}, \hat{\mathbb{P}})$ is a copy of $(\Omega, \mathcal{A}, \mathbb{P})$. It is particularly usefull for 
Let us give some definitions and notation we will need later on about the weighted Ornstein-Uhlenbeck structure on the Wiener space : let $B$ be a standard Brownian motion constructed as coordinates of the space $\mathcal{C}([0,1])$ equipped with the Wiener measure and let $\alpha$ be a positive function in $L_{l o c}^{1}[0,1]$, there exists an error structure (cf. [Bou-03b]) satisfying

$$
\Gamma\left[\int_{0}^{1} u(s) d B_{s}\right]=\int_{0}^{1} \alpha(s) u^{2}(s) d s
$$

for $u \in \mathcal{C}([0,1])$. It is the mathematical expression of the following perturbation of the Brownian path :

$$
\omega(s)=\int_{0}^{s} d B_{u} \mapsto \int_{0}^{s} e^{-\frac{\alpha(u)}{2} \varepsilon} d B_{u}+\int_{0}^{s} \sqrt{1-e^{-\alpha(u) \varepsilon}} d \hat{B}_{u}
$$

where $\hat{B}$ is an independent standard Brownian motion. This structure possesses the following \#-operator :

$$
\left(\int_{0}^{1} u(s) d B_{s}\right)^{\#}=\int_{0}^{1} \sqrt{\alpha(s)} u(s) d \hat{B}_{s}, \quad \forall u \in L^{2}([0,1],(1+\alpha) d t)
$$

which satisfies for regular adapted processes $H$

$$
\left(\int_{0}^{1} H_{s} d B_{s}\right)^{\#}=\int_{0}^{1} \sqrt{\alpha(s)} H_{s} d \hat{B}_{s}+\int_{0}^{1} H_{s}^{\#} d B_{s} .
$$

Let us end this part by a comment on the passage from a random walk to the Brownian motion in the context of erroneous quantities. Donsker's theorem says that if $U_{n}$ are i.i.d. square integrable centered random variables, the linear interpolation of the random walk $\sum_{k=1}^{n} U_{k}$ i.e. the process

$$
X_{n}(t)=\frac{1}{\sqrt{n}}\left(\sum_{k=1}^{[n t]} U_{k}+(n t-[n t]) U_{[n t]+1}\right)
$$

for $t \in[0,1]$, where $[x]$ denotes the entire part of $x$, converges in law on the space $\mathcal{C}([0,1])$ equipped by the uniform norm to a Brownian motion. Invariance principles follow giving a way to approximate properties of the Brownian motion by the corresponding ones of the random walk. A quite natural question is how this may be extended to the case where the $U_{n}$ are erroneous. To extend weak convergence of probability measures we use convergence of Dirichlet forms on Lipschitz and $\mathcal{C}^{1}$ functions. Then supposing the errors on the $U_{n}$ 's are equidistributed and uncorrelated, the error structure of the process $X_{n}$ converges to the Ornstein-Uhlenbeck structure on the Wiener space (cf. [Bou-05a]). Invariance principles follow giving approximations of the variance of the error of Brownian functionals, for example for the sup-norm of the paths :

$$
\mathbb{E} \Gamma\left[\left\|X_{n}(t)\right\|_{\infty}\right]=\mathbb{E} \Gamma\left[\frac{1}{\sqrt{n}} \max _{1 \leq k \leq n}\left|S_{k}\right|\right] \rightarrow \mathbb{E}\left[\int_{0}^{1}\left(D_{s}\left[\|\cdot\|_{\infty}\right]\right)^{2} d s\right]=\mathbb{E}[\mathcal{T}]
$$

where $D$ denotes the Ornstein-Uhlenbeck gradient with values in $L^{2}([0,1])$ and $\mathcal{T}$ is the random time where the absolute value of the Brownian path reaches its maximum.

\section{Propagation of the error due to the Euler scheme.}

If an asset $X$ is represented by the solution of an sde, prices of options, hedging port- 
discovered by Kurtz and Protter in the spirit of a functional central limit theorem takes the form of a process solution to an other sde. In order to propagate this assymptotic error through stochastic calculus, we have to take the derivative in a suitable sense of non differentiable functionals as stochastic integrals. This may be performed by the theory of Dirichlet forms. Let us recall the situation.

\section{The error due to the Euler scheme.}

In 1991 Thomas Kurtz and Philipp Protter obtained an asymptotic estimate in law for the error due to the Euler scheme

In the simplest case, considering the sde

$$
X_{t}=x_{0}+\int_{0}^{t} a\left(X_{s}\right) d B_{s}+\int_{0}^{t} b\left(X_{s}\right) d s
$$

if $X_{t}^{n}$ is the Euler approximation of $X_{t}$ and $U^{n}=X^{n}-X$ then $\left(B, \sqrt{n} U^{n}\right)$ converges in law to $(B, U)$ where $U$ is solution to the linear sde

$$
d U_{t}=a^{\prime}\left(X_{t}\right) U_{t} d B_{t}+b^{\prime}\left(X_{t}\right) U_{t} d t+\frac{1}{\sqrt{2}} a^{\prime}\left(X_{t}\right) a\left(X_{t}\right) d W_{t}, \quad U_{0}=0,
$$

where $W$ is a Brownian motion independent of $B$.

Such an "extra-Brownian motion" appeared in a work of H. Rootzen [Roo-80] who studies limits of integrals of the form $\int_{0}^{t} \psi_{n}(s) d B_{s}$ where $\psi_{n}$ is an adapted process. In the case where $\int_{0}^{t} f\left(B_{s}, s\right) d B_{s}$ is computed by the Euler scheme

$$
\int_{0}^{t} \psi_{n}(s) d B_{s}=\sum_{i=0}^{[n t]} f\left(B_{\frac{i}{n}}, i / n\right)\left(B_{\frac{i+1}{n}}-B_{\frac{i}{n}}\right)+f\left(B_{\frac{[n t]}{n}},[n t] / n\right)\left(B_{t}-B_{\frac{[n t]}{n}}\right)
$$

he obtains for regular $f$

$$
\sqrt{n}\left(\int_{0} \psi_{n} d B-\int_{0}^{\cdot} f\left(B_{s}, s\right) d B_{s}\right) \stackrel{d}{\Rightarrow} \frac{1}{\sqrt{2}} \int_{0}^{\cdot} f_{x}^{\prime}\left(B_{s}, s\right) d W_{s}
$$

This kind of result is restricted to adapted approximations. As Wong and Zakai have shown (1965) other natural approximations of the brownian motion give rise to stochastic integrals in the sense of Stratonowitch

The discovery of the asymptotic error due to the Euler scheme has been followed by a series of works which extend it to the case of an sde with respect to a continuous or discontinuous semi-martingale and which obtain some statements as necessary and sufficient conditions ([Ja-Pr-98], [Ja-Ja-Mé-03]).

In addition, asymptotic expansions have been recently obtained by the stochastic calculus of variation [Ma-Th-03].

In the sequel, we shall consider the result of Kurtz-Protter in dimension 1 under the following form:

Let $X_{t}$ be the solution starting at $x_{0}$ to the sde

$$
d X_{t}=a\left(X_{t}, t\right) d B_{t}+b\left(X_{t}, t\right) d t
$$

let $X_{t}^{n}$ be the approximate solution obtained by the Euler method, which may be written

$$
X_{0}^{n}=x_{0} ; \quad d X_{t}^{n}=a\left(X_{\frac{[n t]}{n}}^{n},[n t] / n\right) d B_{t}+b\left(X_{\frac{[n t]}{n}}^{n},[n t] / n\right) d t
$$

and let $U_{t}^{n}=X_{t}^{n}-X_{t}$ be the approximation error, then if $a$ and $b$ are $\mathcal{C}^{1}$ with linear 
where the process $U$ may be represented as

$$
U_{0}=0 \quad d U_{t}=a_{x}^{\prime}\left(X_{t}, t\right) U_{t} d B_{t}+b_{x}^{\prime}\left(X_{t}, t\right) U_{t} d t+\frac{1}{\sqrt{2}} a_{x}^{\prime}\left(X_{t}, t\right) a\left(X_{t}, t\right) d W_{t}
$$

which is solved by the usual method of variation of the constant : introducing the process

$$
M_{t}=\exp \left\{\int_{0}^{t} a_{x}^{\prime}\left(X_{s}, s\right) d B_{s}-\frac{1}{2} \int_{0}^{t} a_{x}^{\prime 2}\left(X_{s}, s\right) d s+\int_{0}^{t} b_{x}^{\prime}\left(X_{s}, s\right) d s\right\}
$$

gives

$$
U_{t}=M_{t} \int_{0}^{t} \frac{a\left(X_{s}, s\right) a_{x}^{\prime}\left(X_{s}, s\right)}{\sqrt{2} M_{s}} d W_{s} .
$$

Let us consider the weighted Ornstein-Uhlenbeck error structure on the Wiener space with weight $\alpha$ as explain above. If the coefficients $a$ and $b$ are regular, then $X_{t} \in \mathbb{D}$ and $X_{t}^{\#}$ satisfies

(*) $\quad X_{t}^{\#}=\int_{0}^{t} a_{x}^{\prime}\left(X_{s}, s\right) X_{s}^{\#} d B_{s}+\int_{0}^{t} a\left(X_{s}, s\right) \sqrt{\alpha(s)} d \widehat{B_{s}}+\int_{0}^{t} b_{x}^{\prime}\left(X_{s}, s\right) X_{s}^{\#} d s$

Comparing with the equation of the asymptotic error due to the Euler scheme

(**) $\quad U_{t}=\int_{0}^{t} a_{x}^{\prime}\left(X_{s}, s\right) U_{s} d B_{s}+\int_{0}^{t} a\left(X_{s}, s\right) \frac{a_{x}^{\prime}\left(X_{s}, s\right)}{\sqrt{2}} d W_{s}+\int_{0}^{t} b_{x}^{\prime}\left(X_{s}, s\right) U_{s} d s$

shows that

- if we could take a random and adapted weight $\alpha(t)=\frac{1}{2} a_{x}^{\prime 2}\left(X_{t}, t\right)$

- if the obtained structure is closable with carré du champ and if the calculus of the \#-operator is still $(*)$

then $X^{\#}$ would be the asymptotic error due to the Euler scheme, and we would be able to propagate this error through the stochastic computations obtaining the variance of the error on any r. v. $Y \in \mathbb{D}$ by the equation $\Gamma[Y]=\widehat{\mathbb{E}}\left[Y^{\# 2}\right]$.

\section{The Ornstein-Uhlenbeck structure with random weight.}

From now on $\alpha$ is a measurable random process defined on the Wiener space, non negative, non necessarily adapted. We assume that this process satisfies $\mathbb{E} \int_{0}^{1} \alpha_{t} d t<+\infty$, and $\alpha(\omega, t) \geq k(t)>0 \quad \mathbb{P} \times d t$-a.e. where $k$ is deterministic.

Let us denote $\mathbb{D}_{o u}^{k}$ the domain of the Ornstein-Uhlenbeck structure with deterministic weight $k$ and $D_{o u}^{k}$ its gradient. On the domain

$$
\mathbb{D}=\left\{Y \in \mathbb{D}_{\text {ou }}^{k}: \quad \int_{0}^{1} \mathbb{E}\left[\left(D_{\text {ou }}^{k}[Y](t)\right)^{2} \frac{\alpha(t)}{k(t)}\right] d t<+\infty\right\}
$$

which is dense, the form

$$
\mathcal{E}[Y]=\frac{1}{2} \int_{0}^{1} \mathbb{E}\left[\left(D_{\text {ou }}^{k}[Y](t)\right)^{2} \frac{\alpha(t)}{k(t)}\right] d t
$$

is Dirichlet and admits

$$
\Gamma[Y]=\int_{0}^{1}\left(D_{o u}^{k}[Y](t)\right)^{2} \frac{\alpha(t)}{k(t)} d t
$$

as carré du champ operator.

Indeed, let $\mathcal{V}$ be the space of linear combinations of exponentials of the form $Y=$ 
Let $X_{n}$ be a Cauchy sequence in $L^{2}$ and for $\mathcal{E}$. Let $X$ be the limit of $X_{n}$ in $L^{2}$. Then $X_{n}$ is Cauchy for $\mathcal{E}_{o u}^{k}$ which is closed, hence $X \in \mathbb{D}_{o u}^{k}$ and there exists a sub-sequence $X_{n^{\prime}}$ such that

$$
D_{o u}^{k}\left[X_{n^{\prime}}\right] \rightarrow D_{o u}^{k}[X] \quad \mathbb{E} \times d t \text {-p.s. }
$$

and by Fatou's lemma

$$
\begin{gathered}
\int_{0}^{1} \mathbb{E}\left[\left(D_{\text {ou }}^{k}[X]\right)^{2} \frac{\alpha(t)}{k(t)}\right] d t= \\
=\int_{0}^{1} \mathbb{E}\left[\lim \left(D_{\text {ou }}^{k}\left[X_{n^{\prime}}\right]\right)^{2} \frac{\alpha(t)}{k(t)}\right] d t \leq \liminf \int_{0}^{1} \mathbb{E}\left[\left(D_{\text {ou }}^{k}\left[X_{n^{\prime}}\right]\right)^{2} \frac{\alpha(t)}{k(t)}\right] d t<+\infty
\end{gathered}
$$

since $X_{n}$ is Cauchy for $\mathcal{E}$. Hence $X \in \mathbb{D}$. Now again by the Fatou's lemma we show as classically that $X_{n}$ converges to $X$ in $\mathbb{D}$.

Contractions operate on $(\mathcal{E}, \mathbb{D})$ by the functional calculus for $D_{o u}^{k}$ hence $(\mathcal{E}, \mathbb{D})$ is a Dirichlet form. The definition of the carré du champ operator (def 4.1.2 of [Bo-Hi-91]) is satisfied.

The generator $(A, \mathcal{D} A)$ is given by

$$
\begin{aligned}
& \mathcal{D} A=\left\{F \in \mathbb{D} \quad \exists G \in L^{2} \quad \forall H \in \mathbb{D} \quad \frac{1}{2} \mathbb{E} \int_{0}^{1} D_{o u}^{k}[F] D_{\text {ou }}^{k}[H] \frac{\alpha(t)}{k(t)} d t=-<G, H>\right\} \\
& A F=G
\end{aligned}
$$

hence if $F \in \mathcal{D} A$ then $\frac{\alpha(t)}{k(t)} D_{o u}^{k}[F] \in \operatorname{dom} \delta_{o u}^{k}$ and

$$
A F=-\frac{1}{2} \delta_{o u}^{k}\left[\frac{\alpha}{k} D_{o u}^{k} F\right] .
$$

where $\delta_{o u}^{k}$ is the Skorokhod integral with weight $k$.

\section{Adapted case.}

Let us now add the hypothesis that $\alpha$ is adapted. If $h$ is in $L^{\infty}\left(\mathbb{R}_{+}\right)$

$$
\left.\mathbb{E} \Gamma\left[F, \int_{0}^{1} h d B\right]\right]=\mathbb{E}\left[F \int_{0}^{1} h(s) \alpha(s) d B_{s}\right] .
$$

If $F, G \in \mathbb{D} \cap L^{\infty}$

$$
\mathbb{E}[G<D F, h \sqrt{\alpha}>]=-\mathbb{E}[F<D G, h \sqrt{\alpha>}]+\mathbb{E}\left[F G \int h \alpha d B\right] .
$$

And if $v$ is adapted and in $\operatorname{dom} \delta$

$$
\delta[v]=\int_{0}^{1} v_{s} \sqrt{\alpha_{s}} d B_{s} .
$$

At last, for finance, the following properties are important, they use the fact that $\alpha$ is adapted

$$
A\left[\mathbb{E}\left[X \mid \mathcal{F}_{s}\right]\right]=\mathbb{E}\left[A^{s}[X] \mid \mathcal{F}_{s}\right]
$$

where $A^{s}$ is constructed as $A$ with the weight $\alpha(t) 1_{\{t \leq s\}}$,

$$
D\left[\mathbb{E}\left[X \mid \mathcal{F}_{s}\right]\right](t)=\mathbb{E}\left[D[X](t) 1_{t \leq s} \mid \mathcal{F}_{s}\right]
$$

$\mathbb{E}\left[. \mid \mathcal{F}_{s}\right]$ is an orthogonal projector in $\mathbb{D}$ 
where $\#_{s}$ is constructed as $\#$ with the weight $\alpha(t) 1_{\{t \leq s\}}$. If $X$ is $\mathcal{F}_{t}$-measurable, then $A X, \Gamma[X]$ are $\mathcal{F}_{t}$-measurables.

Concerning the operator \# we have the formulae

$$
\left(\int_{0}^{1} \xi_{s} d B_{s}\right)^{\#}=\int_{0}^{1} \xi_{s}^{\#} d B_{s}+\int_{0}^{1} \xi_{s} \sqrt{\alpha_{s}} d \widehat{B}_{s}
$$

Hence formula $(\star)$ is satisfied.

\section{Application to diffusion models.}

Let us consider the following model of an asset

$$
d X_{t}=X_{t} \sigma\left(X_{t}, t\right) d B_{t}+X_{t} r(t) d t
$$

and let us put on the Wiener space the Ornstein-Uhlenbeck structure with weight

$$
\alpha_{t}=\frac{a^{\prime 2}\left(X_{t}, t\right)}{2}=\frac{\left(\sigma\left(X_{t}, t\right)+X_{t} \sigma_{x}^{\prime}\left(X_{t}, t\right)\right)^{2}}{2}
$$

which represents the asymptotic error due to the Euler scheme. $\sigma$ is supposed to be strictly positive, $\mathcal{C}^{1}$ and Lipschitz and the preceding hypotheses on $\alpha$ are assumed.

Such a modelisation is coherent. The error is attached to the asset $X$ and any functional of $X$, including the Brownian motion itself and its error may be computed thanks to the equation

$$
d B_{t}=\frac{d X_{t}}{X_{t} \sigma\left(X_{t}, t\right)}-X_{t} r(t) d t
$$

which gives

$$
\left(B_{t}\right)^{\#}=\int_{0}^{t} \sqrt{\alpha(s)} d \widehat{B}_{s} \quad \Gamma\left[B_{t}\right]=\int_{0}^{t} \alpha(s) d s .
$$

Let us show how financial calculi may be performed before proposing some comments on the use of such an analysis. Puting $M_{t}=\exp \left\{\int_{0}^{t} \sqrt{\alpha_{s}} d B_{s}-\frac{1}{2} \int_{0}^{t} \alpha_{s} d s+\int_{0}^{t} r(s) d s\right\}$ we have

$$
\begin{gathered}
\Gamma\left[X_{t}\right]=M_{t}^{2} \int_{0}^{t} \frac{X_{s}^{2} \sigma^{2}\left(X_{s}, s\right)}{M_{s}^{2}} \alpha_{s} d s \\
\Gamma\left[X_{s}, X_{t}\right]=M_{s} M_{t} \int_{0}^{s \wedge t} \frac{X_{u}^{2} \sigma^{2}\left(X_{u}, u\right)}{M_{u}^{2}} \alpha_{u} d u .
\end{gathered}
$$

The price of a European option with payoff $f\left(X_{T}\right)$ at exercise time $T$

$$
V_{t}=\mathbb{E}\left[\left(\exp -\int_{t}^{T} r(s) d s\right) f\left(X_{T}\right) \mid \mathcal{F}_{t}\right]
$$

becomes erroneous (in the sense of error structures) with an error obtained thanks to the \#:

$$
\begin{gathered}
\Gamma\left[V_{t}\right]=\left(\exp -2 \int_{t}^{T} r(s) d s\right)\left(\mathbb{E}\left[f^{\prime}\left(X_{T}\right) M_{T} \mid \mathcal{F}_{t}\right]\right)^{2} \frac{\Gamma\left[X_{t}\right]}{M_{t}^{2}} \\
\Gamma\left[V_{s}, V_{t}\right]=\left(\exp \left(-\int_{s}^{T} r(u) d u-\int_{t}^{T} r(v) d v\right)\right) \mathbb{E}\left[f^{\prime}\left(X_{T}\right) M_{T} \mid \mathcal{F}_{s}\right] \mathbb{E}\left[f^{\prime}\left(X_{T}\right) M_{T} \mid \mathcal{F}_{t}\right] \frac{\Gamma\left[X_{s}, X_{t}\right]}{M_{s} M_{t}}
\end{gathered}
$$

The quantity of asset in the hedging portfolio is 
and we have

$$
\Gamma\left[H_{t}\right]=\left(\exp -2 \int_{t}^{T} r(s) d s\right)\left(\mathbb{E}\left[\frac{M_{T}}{M_{t}}\left(f^{\prime \prime}\left(X_{T}\right) M_{T}+f^{\prime}\left(X_{T}\right) Z_{t}^{T}\right) \mid \mathcal{F}_{t}\right]\right)^{2} \frac{\Gamma\left[X_{t}\right]}{M_{t}^{2}}
$$

with

$$
\begin{gathered}
Z_{t}^{T}=\int_{t}^{T} L_{s} d B_{s}-\int_{t}^{T} \sqrt{\alpha_{s}} L_{s} M_{s} d s \\
L_{s}=a_{x^{2}}^{\prime \prime}\left(X_{s}, s\right)=2 \sigma_{x}^{\prime}\left(X_{s}, s\right)+X_{s} \sigma_{x^{2}}^{\prime \prime}\left(X_{s}, s\right)
\end{gathered}
$$

It is still true, as in the case of deterministic weight (cf [Bou-03b]), that the proportional error on $X_{t}$ divided by the volatility :

$$
\frac{\sqrt{\Gamma\left[X_{t}\right]}}{X_{t}} \cdot \frac{1}{\sigma\left(X_{t}, t\right)}
$$

is a finite variation process (cf [Ba-Ma-Ma-Re-Th-03]) on the "feed back" effect).

\section{Discussion.}

Thanks to this construction of an error structure, i.e. a local Dirichlet form with squared field operator, on the Wiener space, hence by image, on $\mathcal{C}([0,1])$ equipped with the law of the process $X$, we have at our disposal a powerful mean to propagate the error done on $X$ toward sufficiently smooth functionals of $X$. In order to assess the interest of this tool, the question arises of knowing whether the propagated error is the same as the one we would obtain by a direct computation of the functional thanks to the approximation $X^{n}$ of $X$. For instance, in the simplest case, does the convergence in law

$$
\sqrt{n}\left(f\left(X_{t}^{n}\right)-f\left(X_{t}\right)\right) \stackrel{d}{\Rightarrow} f^{\prime}\left(X_{t}\right) X_{t}^{\#}
$$

hold for $f \in \mathcal{C}^{1} \cap$ Lip ? Can we justify an asymptotic calculus principle which says that the Dirichlet form allows effectively to compute the errors on the quantities which are erroneous because of the approximation $X^{n}$ of $X$ ? We will not exhaustively examine this principle here, for it is a too large enterprise. Nevertheless, in the important current of research whose fruitfulness has been confirmed these last twenty years, which may be called the "tightness programm", the authors, among which we must at least quote P.-A. Meyer, W. A. Zheng, J. Jacod, A. N. Shiryaev, A. Jakubowski, J. Mémin, G. Pagès, T. G. Kurtz, P. Protter, L. Słomiński, D. Talay, V. Bally, A. Kohatsu-Higa and many others, have already done a major part of the work by stating their results of convergence in law, of stable convergence, of tightness of processes, under a sufficiently general form for propagating iteratively the properties through stochastic integrals and sde's in the semi-martingale framework.

Let us give some results in the direction of this asymptotic calculus principle keeping the hypotheses of the present part III.

Let $F$ be a real function of class $\mathcal{C}^{1}$ and Lipschitz defined on $\mathcal{C}([0,1])$ equipped with the uniform norm. Such a function satisfies

$$
F(x+h)=F(x)+<F^{\prime}(x), h>+\|h\| \varepsilon_{x}(h) \quad \forall x, h \in \mathcal{C}([0,1])
$$

where the mapping $x \mapsto F^{\prime}(x)$ is continuous and bounded with values in the Banach space of Radon measures on $[0,1], \varepsilon_{x}(h)$ is bounded in $x$ and $h$, and goes to zero when $h \rightarrow 0$ in $\mathcal{C}([0,1])$. Then we have 
Proof. The equality in the right hand side comes from the functional calculus in error structures (see [Bou-05a]). Puting $U^{n}=X^{n}-X$ as before, the fact that $\sqrt{n}\left\|U^{n}\right\| \varepsilon_{X}\left(U^{n}\right)$ tends to zero in probability, reduces the proof to the study of the convergence in law of

$$
<F^{\prime}(X), \sqrt{n} U^{n}>=\sqrt{n} \int\left(X_{t}^{n}-X_{t}\right) F^{\prime}(X)(d t)
$$

to $\int X_{t}^{\#} F^{\prime}(X)(d t)$. Considering the measure $F^{\prime}(X)(d t)$ as the differential of a finite variation process adapted to the constant filtration $\left.\left.\mathcal{G}_{t}=\mathcal{B}(\mathcal{C}(] 0,1]\right)\right)$, the fact that the process to be integrated $\sqrt{n} U^{n}$ converges stably to $X^{\#}$ implies (cf [Ku-Pr-91b] thm 2.2) that the stochastic integral $\int \sqrt{n} U^{n} F^{\prime}(X)(d t)$ converges in law to $\int X_{t}^{\#} F^{\prime}(X)(d t)$.

We obtain also the convergence in law of the stochastic integrals $H \cdot \sqrt{n} U^{n} \stackrel{d}{\Rightarrow} H . X^{\#}$ for $H$ deterministic or adapted and that of

$$
\sqrt{n}\left(\int_{0}^{1} f\left(X_{s}^{n}, s\right) d X_{s}^{n}-\int_{0}^{1} f\left(X_{s}, s\right) d X_{s}\right)
$$

to

$$
\left(\int_{0}^{1} f\left(X_{s}, s\right) d X_{s}\right)^{\#}=\int_{0}^{1} f^{\prime}\left(X_{s}, s\right) X_{s}^{\#} d X_{s}+\int_{0}^{1} f\left(X_{s}, s\right) d X_{s}^{\#}
$$

for $f \mathcal{C}^{1}$ and Lipschitz.

More generally, we can make more explicit the research programm of determining the domain of the asymptotic calculus.

Let $X_{n}$ and $X$ be two random variables with values in a measurable set $(E, \mathcal{F})$, and let $\alpha_{n}$ be a sequence of positive numbers. Let $\mathcal{D}_{0}$ denote a set of simple functions included in $L^{2}\left(\mathbb{P}_{X}\right)$ and in $L^{2}\left(\mathbb{P}_{X_{n}}\right) \forall n$. Let us suppose that there exists an error structure

$$
S=\left(E, \mathcal{F}, \mathbb{P}_{X}, \mathbb{D}, \Gamma\right)
$$

such that $\mathcal{D}_{0} \subset \mathbb{D}$ and $\forall \varphi \in \mathcal{D}_{0}$

$$
\lim _{n} \alpha_{n} \mathbb{E}\left[\left(\varphi\left(X_{n}\right)-\varphi(X)\right)^{2}\right]=\mathbb{E}[\Gamma[\varphi]]
$$

we shall say that the asymptotic calculus principle extends to $\mathcal{D}$ for $\mathcal{D}_{0} \subset \mathcal{D} \subset \mathbb{D}$ if the limit (1) extends to $\psi \in \mathcal{D}$.

If, as above, a \#-operator is available (which occurs as soon as $\mathbb{D}$ is separable), in order to prove $(1)$ on $\mathcal{D}$, since \# is a closed operator, it suffices for any $\psi \in \mathcal{D}$ to find a sequence $\varphi_{p} \in \mathcal{D}_{0}$ such that

i) $\varphi_{p} \rightarrow \psi$ in $L^{2}\left(\mathbb{P}_{X}\right)$

ii) $\varphi_{p}^{\#}$ converges in $L^{2}\left(\mathbb{P}_{X} \times \widehat{\mathbb{P}_{X}}\right)$

iii) $\left.\quad \alpha_{n} \mathbb{E}\left[\psi\left(X_{n}\right)-\psi(X)\right)^{2}\right]$ may be approximated uniformly in $n$ by $\left.\alpha_{n} \mathbb{E}\left[\varphi_{p}\left(X_{n}\right)-\varphi_{p}(X)\right)^{2}\right]$.

When $(E, \mathcal{F})$ is a normed vectorspace, obtaining $(1)$ from a convergence in law of $\sqrt{\alpha_{n}}\left(\varphi\left(X_{n}\right)-\right.$ $\varphi(X))$ uses generally a uniform integrability of $\alpha_{n}\left\|X_{n}-X\right\|^{2}$. We shall go deaper in this problem in a separate work.

Let us end by some remarks from the point of view of finance. The interest of considering a financial asset as erroneous is not evident since it is one of the best known quantities 
bid-ask. But this would rather justify specifically constructed error structures instead of the one induced by the Euler scheme. This error structure is relevant only in order to assess the errors in Monte Carlo simulations performed to calculate financial quantities in a given model.

Several authors ([Du-Pr-89], [Ha-My-05]) remarked that the stochastic integral which is the active hedge of a future contingent claim, in a model where the underlying asset is a semi-martingale, is an instance of application of limit theorems on discretization errors. This is different from the Euler scheme error and it would be worth to examine this error from the point of view of an asymptotic Dirichlet form.

A more general and complete study of the bias operators and the Dirichlet form yielded by an approximation, with applications related to the part III of the present paper, is to appear, ([Bou-06]).

\section{REFERENCES}

[Al-Ma-Nu-00] Alos E., Mazet O., Nualart D. "Stochastic calculus with respect to fractional Brownian motion with Hurst parameter lesser than 1/2" Stochastic Process. Appl. 86, (2000).

[Ba-Ta-96a] BAlly V., TAlay D. "The law of the Euler scheme for stochastic differential equations: I. Convergence rate of the distribution function", Prob. Th. and Rel. Fields vol 2 No2, 93-128 (1996)

[Ba-Ta-96b] Bally V., TAlay D. "The law of the Euler scheme for stochastic differential equations : II. Convergence rate of the density", Monte Carlo Methods and Appl. vol 104, No1, 43-80 (1996)

[Ba-Ma-Ma-Re-Th-03] Barucci E., Malliavin P., Mancino M.E., Renó R., ThalMAIER A. "The price volatility feedback rate: an implementable indicator of market stability" Math. Finance, 13, 17-35, 2003.

[Bo-Ek-To-04] Bouchard B., Ekeland I., Touzi N. "On the Malliavin approach to Monte Carlo approximation of conditional expectations", Finance Stoch. 8, 45-71, (2004),

[Bou-85] Bouleau N. "About stochastic integrals with respect to processes which are not semi-martingales" Osaka J. Math. 22, 31-34, (1985),

[Bou-03a] Bouleau N. "Error calculus and path sensitivity in Financial models", Mathematical Finance vol 13/1, 115-134, (2003).

[Bou-03b] Bouleau N. Error Calculus for Finance and Physics, the Language of Dirichlet Forms, De Gruyter, 2003.

[Bou-04] Bouleau N. Financial Markets and Martingales, Observations on Science and Speculation, Springer 2004.

[Bou-05a] Bouleau N. "Théorème de Donsker et formes de Dirichlet" Bull. Sci. Math. 129, (2005), 369-380.

[Bou-05b] Bouleau N. "Improving Monte Carlo simulations by Dirichlet forms" C. R. Acad. Sci. Paris Ser I (2005)

[Bou-06] Bouleau N. "When and How an error yields a Dirichlet form" J. of Functional Analysis, to appear.

[Bo-Hi-91] Bouleau N., Hirsch F. Dirichlet Forms and Analysis on Wiener Space, De Gruyter, (1991).

[Ca-Fe-Nu-98] Caballero M. E., Fernandez B., Nualard, D. "Estimation of densities and applications" J. of Theoretical Prob. 11, No3, (1998),

[De-Gr-Po-99] Denis L., Grorud A., Pontier M. "Formes de Dirichlet sur un espace 
[Du-Pr-89] Duffie D., Protter P., "From discrete to continuous time finance : weak convergence of the financial gain process" (1989) (unpublished, cited in [Ku-Pr-91b])

[El-Pr-04] El Kahtib Y., Privault N. "Computation of Greeks in a market with jumps via Malliavin calculus" Finance and Stoch. 8, 161-179, (2004),

[Eme-89] EMERY M. Stochastic calculus on Manifolds Springer (1989),

[Er-Ru-98] Errami M., Russo F. "Covariation de convolution de martingales" $C$. $R$. Acad. Sci. Paris, s1, 326, 601-609, (1998),

[Fe-LaP-99] Feyel D., La Pradelle A. De, "On fractional Brownian processes" Potential Anal. 10(3), 273-288, (1999),

[Fl-Ru-Wo-00] Flandoli F., Russo F., Wolf J. "Some SDEs with distributional drift, I. General calculus" Osaka J. Math. 40, No2, 493-542, (2003).

[Föl-80] Föllmer H. "Dirichlet processes" in Stochastic Integrals, lect. Notes in Math. No 851, 476-478, (1981).

[Föl-81] Föllmer H. "Calcul d'Ito sans probabilité" in Sém. Prob. XV, Lect. N. in Math. 850, 143-150, Springer (1981),

[Fö-Pr-Sh-95] Föllmer H., Protter P., Shiryaev A. N. "Quadratic covariation and an extension of Ito's formula" Bernoulli 1, 149-169, (1995),

[Fo-La-Le-Li-To-99] Fournié E., Lasry J. M., Lebuchoux J., Lions P. L., Touzi N. "Applications of Malliavin calculus to Monte Carlo methods in finance" Finance and Stoch. 3, 391-412, (1999),

[Fo-La-Le-Li-01] Fournié E., Lasry J. M., Lebuchoux J., Lions P. L. "Applications of Malliavin calculus to Monte Carlo methods in finance II" Finance and Stoch.(2001),

[Fuk-80] Fukushima M. Dirichlet forms and Markov processes, North-Holland (1980),

[Fu-Os-Ta-94] Fukushima M., Oshima Y., Takeda M. Dirichlet forms and symmetric Markov processes, De Gruyter 1994.

[Go-Ko-01] Gobet E., Kohatsu-Higa A. "Computation of Greeks for barrier and lookback options using Malliavin calculus" Electron. Com. in Prob. 8, 51-62, (2003).

[Gr-No-03] Gradinaru M., Nourdin I. "Approximation at first and second order of the $m$-variation of the fractional Brownian motion" Electron. Com. Prob. 8, 1-26, (2003)

[Gr-Ru-Va-03] Gradinaru M., Russo F., Vallois P. "Generalized covariation, local time and Stratonowich-Ito's formula for fractional Brownian motion with Hurst index $\geq$ 1/4" Annals of Prob. 31, No4, 1772-1820, (2003).

[Ha-My-05] Hayashi T., Mykland P.A. "Evaluating hedging errors : an asymptotic approach" Math. Finance, vol 15, No 2, 309-343, (2005)

[Jac-85] JACOD J., "Théorèmes limites pour les processus" Lect. Notes Math. vol 1117, Springer 1985.

[Ja-Ja-Mé-03] Jacod J., Jakubowski A., MÉmin J. "About asymptotic errors in discretization processes" Ann; of Prob.31, 592-608, (2003),

[Ja-Pr-98] Jacod J., Protter P. "Asymptotic error distributions for the Euler method for stochastic differential equations" Ann. Probab. 26, 267-307, (1998)

[Ja-Sh-87] Jacod J., ShIRYAev A.N., Limit Theorems for Stochastic Processes, Springer, 1987.

[Ja-Mé-Pa-89] Jakubowski A., Mémin J., PAGÈs G. "Convergence en loi des suites d'intégrales stochastiques sur l'espace de Skorokhod" Probab. Th. Rel. Fields 81, 111-137, 1989.

[Ko-Pe-02] Kohatsu-Higa A., Pettersson R. "Variance reduction methods for simulation of densities on Wiener space", SIAM J. Numer. Anal. Vol 40, No2, 431-450, (2002) 
simulation schemes for SDEs" Stochastic Analysis 331-346, Acad. Press, 1991.

[Ku-Pr-91b] Kurtz, T.; Protter, Ph. "Weak limit theorems for stochastic integrals ans stochastic differential equations" Ann. Probab. 19, 1035-1070, 1991.

[Le-Na-Nu-03] Leon J. A., Navarro R., Nualart D. "An anticipating calculus approach to the utility maximization of an insider" Math. Finance 13, No1, 171-185, (2003).

[LeJ-78] LE JAN Y. "Mesures associées à une forme de Dirichlet, applications" Bull. Soc. Math. France 106, 61-112, (1978),

[Ly-Zh-98] Lyons T., Zheng W. "A crossing estimate for the canonical process on a Dirichlet space and tightness result" in Colloque Paul Lévy, Astérisque No 157-158, pp 249-271, (1998),

[Ma-Rö-92] MA Z. M., RöckneR M. Introduction to the Theory of (Non-Symmetric) Dirichlet Forms Springer (1992),

[Ma-Th-03] Malliavin P., Thalmaier A. "Numerical error for SDE: Asymptotic expansion and hyperdistributions", C. R. Acad. Sci. Paris ser. I 336 (2003) 851-856

[Ma-Th-05] Malliavin P., Thalmaier A. Stochastic Calculus of Variations in Mathematical Finance, Springer, (to appear 2005),

[Me-Pr-03] Mensi M., Privault N. "Conditional calculus and enlargement of filtration on Poisson space" Stoch. Anal. and Appl. 21, 183-204,(2003),

[Mey-80] Meyer P.-A. "Géométrie différentielle stochastique" in Sém. Prob. XVI suppl. 165-207, Lect. N. in Math. 921, Springer (1982),

[Nua-95] Nualart N. The Malliavin calculus and related topics. Springer, 1995.

[Osh-92] Oshima, Y "On a construction of Markov processes associated with time dependent Dirichlet spaces" Forum Math. No 4, 395-415, (1992).

[Roo-80] RootzÉn, H. "Limit distribution for the error in approximation of stochastic integrals" Ann. Probab. 8, 241-251, (1980).

[Ru-Va-95] Russo F., VAllois P. "The generalized covariation process and Itô formula" Stochastic Proc. Appl. 59, 81-104, (1995),

[Ru-Va-96] Russo F., Vallois P. "Itô formula for $C^{1}$-functions of semi-martingales" Prob. Th. Rel. Fields 104, 27-41, (1996),

[Ru-Va-00] Russo F., Vallois P. "Stochastic calculus with respect to a finite quadratic variation process" Stochastics and Stoch. Rep. 70, 1-40, (2000),

[Ru-Va-Wo-01] Russo F., Vallois P., Wolf J. "A generalized class of Lyons-Zheng processes" Bernoulli 7, No2, 363-379, (2001),

[Sch-82] SchWARTz L. "Géométrie différentielle du 2ème ordre, semi-martingales et équations différentielles stochastiques sur une variété différentielle" in Sém. Prob. XVI suppl. 1-150, Lect. N. in Math. 921, Springer (1982),

[Sło-89] SŁOmiński, L. "Stability of strong solutions of stochastic differential equations" Stochastic Process. Appl. 31, 173-202, (1989).

[Sta-99] Stannat, W. "The theory of generalized Dirichlet forms and its applications in analysis and stochastics" Mem. Amer. Math. Soc. 142, No 678, (1999).

[Tru-00] Trutnau G. "Stochastic calculus of generalized Dirichlet forms and applications to stochastic differential equations in infinite dimensions" Osaka J. Math. 37(2), 315$343,(2000)$,

[Wol-97] Wolf J. "An Ito formula for Dirichlet processes" Stochastics and Stoch. Rep. 62(2), 103-115, (1997),

[Wo-Za-65] Wong E., ZAKAi M. "On the convergence of ordinary integrals to stochastic integrals" Ann. Math. Statist. 36, 1560-1564, (1965)

[Zah-98] ZAHLE M. "Integration with respect to fractal functions and stochastic calculus" 